\title{
Soft-wall model of AdS/QCD: The case of light scalar mesons
}

\author{
Fulvia De Fazio \\ Istituto Nazionale di Fisica Nucleare, Sezione di Bari, Italy \\ E-mail: fulvia.defazio@ba.infn.it
}

\begin{abstract}
We study light scalar mesons in the AdS/QCD soft-wall model with a background dilaton field, to investigate the features of this approach in describing QCD properties in the strong coupling regime. We find that the masses and decay constants are compatible with experiment and QCD determinations if $a_{0}(980)$ and $f_{0}(980)$ are identified as the lightest scalar mesons; moreover, the states are organized in linear Regge trajectories with the same slope of vector mesons. Strong couplings of scalar states to pairs of light pseudoscalar mesons turn out to be small, at odds with experiment and QCD estimates: this discrepancy is related to the description of chiral symmetry breaking in this holographic model.
\end{abstract}

8th Conference Quark Confinement and the Hadron Spectrum

September 1-6, 2008

Mainz. Germany 
The AdS/CFT correspondence was proposed by Maldacena [1] as a duality between a type IIB string theory defined on $\mathrm{AdS}_{5} \times S^{5}$ space $\left(\mathrm{AdS}_{5}\right.$ being a 5 dimensional Anti de Sitter space and $S^{5}$ the $5 \mathrm{~d}$ sphere) and a $\mathscr{N}=4$ super Yang-Mills theory with gauge group $S U\left(N_{c}\right)$, for large $N_{c}$. Later on, it was supposed that the correspondence could be generalized as an equivalence between a theory defined on $\operatorname{AdS}_{d+1} \times \mathscr{C}(\mathscr{C}$ being a compact manifold $)$ and a conformal field theory living on the flat boundary $\mathscr{M}_{d}$ of the AdS space [2]. This has provided new hints on the possibility of describing strong interaction processes by string-inspired approaches. Two main ways are followed to achieve such a result. The first one, the so-called top-down approach, consists in starting from a string theory trying to derive a low-energy QCD-like theory on $\mathscr{M}_{d}$ through compactifications of the extra dimensions [3]. In the second one, the bottom-up approach, one starts from $4 d$ QCD and attempts to construct its higher dimensional dual theory [4], with phenomenological properties as guidelines.

In both approaches it is necessary to break conformal invariance, since QCD is not a conformal theory [5], and to account for confinement. In the bottom-up approach, one possibility (hard-wall model) is to use a five dimensional "AdS-slice" with the fifth (holographic) coordinate $z$ varying up to $z_{\max }$ of $\mathscr{O}\left(\frac{1}{\Lambda_{Q C D}}\right)[4,6]$. Another proposal to break conformal invariance consists in introducing in the $5 d$ AdS space a background dilaton field (soft-wall model) [7, 8].

Here we report an analysis of light scalar mesons in the soft wall model [9]. In particular, we describe the derivation of the mass spectrum, the decay constants and the strong couplings of scalar mesons to pairs of light pseudoscalars. The comparison of the results obtained in the AdS framework with experiment and QCD calculations can shed light on the features and drawbacks of the model. Other analyses of scalar mesons in holographic approaches can be found in $[10,11]$.

\section{The model}

In the $5 d$ space we consider the metric: $d s^{2}=g_{M N} d x^{M} d x^{N}=\frac{R^{2}}{z^{2}}\left(\eta_{\mu v} d x^{\mu} d x^{v}+d z^{2}\right)$ with $\eta_{\mu \nu}=\operatorname{diag}(-1,+1,+1,+1) ; R$ is the AdS curvature radius and the holographic coordinate $z$ runs from zero to infinity. The model is characterized by a background dilaton field: $\Phi(z)=(c z)^{2}$, the form of which is chosen to obtain light vector mesons with linear Regge trajectories [8]; $c$ is a dimensionful parameter setting the scale of QCD quantities. We consider the $5 d$ action:

$$
S_{\text {eff }}=-\frac{1}{k} \int d^{5} x \sqrt{-g} e^{-\Phi(z)} \operatorname{Tr}\left\{|D X|^{2}+m_{5}^{2} X^{2}+\frac{1}{2 g_{5}^{2}}\left(F_{V}^{2}+F_{A}^{2}\right)\right\}
$$

where $g$ is the determinant of the metric tensor $g_{M N}$ and $k$ a dimensionful parameter providing a dimensionless action. This action includes fields dual to QCD operators defined at the boundary $z=0$. There is a scalar bulk field $X$, whose mass is fixed by the AdS/CFT relation: $m_{5}^{2} R^{2}=(\Delta-$ $p)(\Delta+p-4), \Delta$ being the dimension of the $p-$ form QCD operator dual to $X$. This field, written as $X=\left(X_{0}+S\right) e^{2 i \pi}$, contains a field $X_{0}(z)=\frac{v(z)}{2}$, the scalar field $S(x, z)$ and the chiral field $\pi(x, z)$. $X_{0}$ is dual to $\langle\bar{q} q\rangle$ and is responsible for chiral symmetry breaking. $S$ includes singlet $S_{1}(x, z)$ and octet $S_{8}^{a}(x, z)$ components: $S=S^{A} T^{A}=S_{1} T^{0}+S_{8}^{a} T^{a}$ with $T^{0}=1 / \sqrt{2 n_{F}}$ and $T^{a}$ the generators of $S U(3)_{F}(A=0, a$, and $a=1, \ldots 8) . S^{A}$ is dual to $\mathscr{O}_{S}^{A}(x)=\bar{q}(x) T^{A} q(x)$, so that $\Delta=3, p=0$ and $m_{5}^{2} R^{2}=-3$. The fields $A_{L, R}^{a}(x, z)$ are introduced to gauge the chiral symmetry in the $5 d$ space. They are dual to $\bar{q}_{L, R} \gamma_{\mu} T^{a} q_{L, R}$ and can be written in terms of vector $V$ and axial-vector $A$ fields: 
$V^{M}=\frac{1}{2}\left(A_{L}^{M}+A_{R}^{M}\right), A^{M}=\frac{1}{2}\left(A_{L}^{M}-A_{R}^{M}\right)$, so that $F_{V}^{M N}=\partial^{M} V^{N}-\partial^{N} V^{M}-i\left[V^{M}, V^{N}\right]-i\left[A^{M}, A^{N}\right]$, $F_{A}^{M N}=\partial^{M} A^{N}-\partial^{N} A^{M}-i\left[V^{M}, A^{N}\right]-i\left[A^{M}, V^{N}\right]$ and $D^{M} X=\partial^{M} X-i\left[V^{M}, X\right]-i\left\{A^{M}, X\right\}$.

Using $S_{\text {eff }}$ in (1.1), we assume that the AdS/CFT duality relation: $\left\langle e^{i \int d^{4} x \mathscr{O}(x) f_{0}(x)}\right\rangle_{Q C D}=e^{i S_{e f f}}$ holds, where the lhs is the QCD generating functional in which the sources $f_{0}(x)$ of the $4 d \mathscr{O}(x)$ operators are the boundary $(z \rightarrow 0)$ limits of the corresponding (dual) $5 d$ fields. We then derive the properties of light scalar mesons in the soft-wall model.

\section{Spectrum of scalar mesons}

Let us consider the quadratic part of the action (1.1) involving the scalar fields $S^{A}(x, z)$ :

$$
S_{\text {eff }}^{(2)}=-\frac{1}{2 k} \int d^{5} x \sqrt{-g} e^{-\Phi(z)}\left(g^{M N} \partial_{M} S^{A} \partial_{N} S^{A}+m_{5}^{2} S^{A} S^{A}\right) .
$$

From this term, we derive the equation of motion for the field $S^{A}$ :

$$
\eta^{M N} \partial_{M}\left(\frac{R^{3}}{z^{3}} e^{-\Phi(z)} \partial_{N} S\right)+3 \frac{R^{3}}{z^{5}} e^{-\Phi(z)} S=0
$$

where we dropped the flavour index $A$. We identify scalar meson states with the normalizable solutions of this equation corresponding to the discrete mass spectrum [11]: $-q_{n}^{2}=m_{n}^{2}=c^{2}(4 n+6)$ with integer $n$, and eigenfunctions expressed in terms of the generalized Laguerre polynomials $\tilde{S}_{n}(\hat{z})=\sqrt{\frac{2}{n+1}} \hat{z}^{3} L_{n}^{1}\left(\hat{z}^{2}\right)$ (we have defined $S(x, z)=\int \frac{d^{4} q}{(2 \pi)^{4}} e^{i q \cdot x} \tilde{S}(q, z)$ ).

Scalar mesons are organized in linear Regge trajectories with the same slope as vector mesons and scalar glueballs, the spectral condition of which is $m_{\rho_{n}}^{2}=c^{2}(4 n+4)[8]$ and $m_{G_{n}}^{2}=c^{2}(4 n+8)$ [12], respectively, with the parameter $c$ setting the scale of all hadron masses.

Scalar mesons turn out to be heavier than vector mesons. This is in quantitative agreement with experiment if $a_{0}(980)$ and $f_{0}(980)$ are identified as the lightest scalar mesons, since the results in $\operatorname{AdS} R_{f_{0}\left(a_{0}\right)}=\frac{m_{f_{0}\left(a_{0}\right)}^{2}}{m_{\rho^{0}}^{2}}=\frac{3}{2}$ agree with $R_{f_{0}}^{\text {exp }}=1.597 \pm 0.033$ and $R_{a_{0}}^{\text {exp }}=1.612 \pm 0.004$. Finally, scalar mesons are lighter than scalar glueballs: $\frac{m_{G}^{2}}{m_{f_{0}}^{2}}=\frac{4}{3}$ for the lowest-lying states.

\section{Two-point correlation function of the scalar operator}

Let us consider in QCD the two-point correlation function:

$$
\Pi_{Q C D}^{A B}\left(q^{2}\right)=i \int d^{4} x e^{i q \cdot x}\left\langle 0\left|T\left[\mathscr{O}_{S}^{A}(x) \mathscr{O}_{S}^{B}(0)\right]\right| 0\right\rangle
$$

with $\mathscr{O}_{S}^{A}(x)=\bar{q}(x) T^{A} q(x)$. The AdS/CFT method relates this correlation function to the two-point correlator obtained from the action (1.1): $\Pi_{A d S}^{A B}\left(q^{2}\right)=\left.\delta^{A B} \frac{R^{3} c^{4}}{k} S\left(\frac{q^{2}}{c^{2}}, \hat{z}^{2}\right) \frac{e^{-\Phi(\hat{z})}}{\hat{z}^{3}} \partial_{\hat{z}} S\left(\frac{q^{2}}{c^{2}}, \hat{z}^{2}\right)\right|_{\hat{z} \rightarrow 0}$ where the bulk-to-boundary propagator $\widetilde{S}\left(q^{2}, z\right)=S\left(q^{2} / c^{2}, \hat{z}^{2}\right) \widetilde{S}_{0}\left(q^{2}\right)$ is obtained solving eq. (2.2) for all four-momenta $q^{2}$. We get:

$$
\begin{aligned}
\Pi_{A d S}^{A B}\left(q^{2}\right) & =\delta^{A B} \frac{4 c^{2} R}{k}\left[\left(\frac{q^{2}}{4 c^{2}}+\frac{1}{2}\right) \ln \left(c^{2} z^{2}\right)+\left(\gamma_{E}-\frac{1}{2}\right)+\frac{q^{2}}{4 c^{2}}\left(2 \gamma_{E}-\frac{1}{2}\right)\right. \\
& \left.+\left(\frac{q^{2}}{4 c^{2}}+\frac{1}{2}\right) \psi\left(\frac{q^{2}}{4 c^{2}}+\frac{3}{2}\right)\right]\left.\right|_{z=z_{\min }}
\end{aligned}
$$


(omitting a $\mathscr{O}\left(\frac{1}{z^{2}}\right)$ contact term). This expression shows the presence of a discrete set of poles, those the Euler function $\psi$, with masses $m_{n}^{2}=c^{2}(4 n+6)$ and residues $F_{n}^{2}=\frac{R}{k} 16 c^{4}(n+1)$. The factor $\frac{R}{k}$ can be fixed by matching (3.2) in the $q^{2} \rightarrow+\infty$ (i.e. in the short-distance) limit, expanded in powers of $1 / q^{2}$, with the QCD result, giving: $\frac{R}{k}=\frac{N_{c}}{16 \pi^{2}}$. The residues of the two-point correlator, related to the scalar meson decay constants, are now determined: $F_{n}^{2}=\frac{N_{c}}{\pi^{2}} c^{4}(n+1)$.

We compare this result to QCD calculations. For $a_{0}(980)$, the following result was obtained $F_{a_{0}}=\left\langle 0\left|\mathscr{O}_{S}^{3}\right| a_{0}(980)^{0}\right\rangle=(0.21 \pm 0.05) \mathrm{GeV}^{2}$ [13]. The AdS prediction is: $F_{a_{0}}=\frac{\sqrt{3}}{\pi} c^{2}=$ $0.08 \mathrm{GeV}^{2}$, having fixed $c$ from the $\rho^{0}$ mass: $c=\frac{m_{\rho}}{2}$. For the $f_{0}(980)$ a similar result was obtained for the matrix element of the $s \bar{s}$ operator: $\left\langle 0|\bar{s} s| f_{0}(980)\right\rangle=(0.18 \pm 0.015) \mathrm{GeV}^{2}$ [14]. Considering the uncertainties in QCD determinations, the AdS results differ by about a factor of two.

\section{Interaction of scalar mesons with a pair of pseudoscalar mesons}

In (1.1) the interaction terms involving one scalar $S$ and two light pseudoscalar fields $P$ only appear in the term $\operatorname{Tr}\left\{|D X|^{2}\right\}$. Using the equations of motion and writing the axial-vector bulk field in terms of the transverse and longitudinal components: $A_{M}=A_{\perp M}+\partial_{M} \phi$, we have:

$$
S_{\text {eff }}^{(S P P)}=-\frac{4}{k} \int d^{5} x \sqrt{-g} e^{-\Phi(z)} g^{M N} v(z) \operatorname{Tr}\left\{S\left(\partial_{M} \pi-\partial_{M} \phi\right)\left(\partial_{N} \pi-\partial_{N} \phi\right)\right\} .
$$

Defining $\psi^{a}=\phi^{a}-\pi^{a}$ and for $n_{F}=3$ we have:

$$
S_{\text {eff }}^{(S P P)}=-\frac{R^{3}}{k} \int d^{5} x \frac{1}{z^{3}} e^{-\Phi(z)} v(z)\left[\frac{2}{\sqrt{6}} S_{1} \eta^{M N}\left(\partial_{M} \psi^{a}\right)\left(\partial_{N} \psi^{a}\right)+d^{a b c} S_{8}^{a} \eta^{M N}\left(\partial_{M} \psi^{b}\right)\left(\partial_{N} \psi^{c}\right)\right] \text {. }
$$

If the fields are expressed in terms of their bulk-to-boundary propagators and of the corresponding sources, the three point function involving two pseudoscalar and one scalar operator can be obtained by functional derivation of the action with respect to the source fields. The AdS result can be compared with the QCD three-point function:

$$
\Pi_{Q C D \alpha \beta}^{a b c}\left(p_{1}, p_{2}\right)=i^{2} \int d^{4} x_{1} d^{4} x_{2} e^{i p_{1} \cdot x_{1}} e^{i p_{2} \cdot x_{2}}\left\langle 0\left|T\left[\mathscr{O}_{5_{\alpha}}^{b}\left(x_{1}\right) \mathscr{O}_{S}^{a}(0) \mathscr{O}_{5_{\beta}}^{c}\left(x_{2}\right)\right]\right| 0\right\rangle .
$$

Since $\Pi_{Q C D \alpha \beta}^{a b c}\left(p_{1}, p_{2}\right)$ can be written in terms of the coupling $g_{S_{n} P P}$ as follows:

$$
\Pi_{Q C D \alpha \beta}^{a b c}\left(p_{1}, p_{2}\right)=d^{a b c} \frac{p_{1 \alpha} p_{2 \beta}}{p_{1}^{2} p_{2}^{2}} f_{\pi}^{2} \sum_{n=0}^{\infty} \frac{F_{n} g_{S_{n} P P}}{q^{2}+m_{n}^{2}}
$$

with $q=-\left(p_{1}+p_{2}\right)$ and $f_{\pi}$ the pion decay constant, comparison with the expression obtained in AdS allows to determine $g_{S_{n} P P}$. For the lowest radial number $n=0$ we have:

$$
g_{S_{0} P P}=\frac{\sqrt{N_{c}}}{4 \pi} \frac{m_{S_{0}}^{2}}{f_{\pi}^{2}} R c \int_{0}^{\infty} d \hat{z} e^{-\hat{z}^{2}} v(\hat{z})
$$

with $\hat{z}=c z . g_{S_{0} P P}$ depends linearly on the field $v(z)$ introduced in Section 1, which can be obtained solving the equation of motion which stems from the action (1.1). The numerical result is small, of $\mathscr{O}(10) \mathrm{MeV}$ depending on the input quark mass, while phenomenological determinations of $g_{S_{0} P P}$ indicate sizeable values. For example the experimental value of $g_{a_{0}} \eta \pi$ is: $g_{a_{0}} \eta \pi=12 \pm 6 \mathrm{GeV}$, while for $f_{0}$ a QCD estimate gives: $g_{f_{0} K^{+} K^{-}} \simeq 6-8 \mathrm{GeV}$ [15]. The origin of the small value for the $S P P$ couplings in the soft-wall model is related to the difficulty of correctly reproducing chiral symmetry breaking in this model through the non vanishing chiral condensate and light quark masses $[8,9]$. 


\section{Conclusions}

In the AdS soft-wall model the masses of scalar meson are close to experiment, but their decay constants differ from the available QCD determinations by about a factor of two. The strong couplings $g_{S P P}$ are smaller than in phenomenological determinations; this is related to a difficulty in precisely describing chiral symmetry breaking within this model.

\section{Acknowledgments}

I thank P. Colangelo, F. Giannuzzi, F. Jugeau and S. Nicotri for collaboration. This work was supported in part by the EU Contract No. MRTN-CT-2006-035482, "FLAVIAnet".

\section{References}

[1] J. M. Maldacena, The large N limit of superconformal field theories and supergravity, Adv. Theor. Math. Phys. 2 (1998) 231 [Int. J. Theor. Phys. 38 (1999) 1113].

[2] E. Witten, Anti-de Sitter space and holography, Adv. Theor. Math. Phys. 2 (1998) 253; Anti-de Sitter space, thermal phase transition, and confinement in gauge theories, ibidem 2 (1998) 505; S. S. Gubser et al., Gauge theory correlators from non-critical string theory, Phys. Lett. B 428 (1998) 105.

[3] For a recent review see: J. Erdmenger, N. Evans, I. Kirsch and E. Threlfall, Mesons in Gauge/Gravity Duals - A Review, Eur. Phys. J. A 35 (2008) 81 and references therein.

[4] J. Polchinski et al., Hard scattering and gauge/string duality, Phys. Rev. Lett. 88 (2002) 031601.

[5] For a discussion see S. J. Brodsky and G. F. de Teramond, arXiv:0802.0514.

[6] J. Erlich, E. Katz, D. T. Son and M. A. Stephanov, QCD and a holographic model of hadrons, Phys. Rev. Lett. 95 (2005) 261602; L. Da Rold and A. Pomarol, Chiral symmetry breaking from five dimensional spaces, Nucl. Phys. B 721 (2005) 79.

[7] O. Andreev, 1/q2 corrections and gauge / string duality, Phys. Rev. D 73 (2006) 107901.

[8] A. Karch et al., Linear confinement and AdS/QCD, Phys. Rev. D 74 (2006) 015005.

[9] P. Colangelo, F. De Fazio, F. Giannuzzi, F. Jugeau and S. Nicotri, Light scalar mesons in the soft-wall model of AdS/QCD, Phys. Rev. D 78 (2008) 055009.

[10] K. Ghoroku, N. Maru, M. Tachibana and M. Yahiro, Holographic model for hadrons in deformed AdS(5) background, Phys. Lett. B 633, 602 (2006); L. Da Rold and A. Pomarol, The scalar and pseudoscalar sector in a five-dimensional approach to chiral symmetry breaking, JHEP 0601(2006) 157; H. Forkel, M. Beyer and T. Frederico, Linear square-mass trajectories of radially and orbitally excited hadrons in holographic QCD, JHEP 0707 (2007) 077; T. Huang and F. Zuo, Couplings of the Rho Meson in a Holographic dual of QCD with Regge Trajectories, Eur. Phys. J. C 56 (2008) 75.

[11] A. Vega and I. Schmidt, Scalar hadrons in $A d S_{5} \times S^{5}$, Phys. Rev. D 78 (2008) 017703.

[12] P. Colangelo, F. De Fazio, F. Jugeau and S. Nicotri, On the light glueball spectrum in a holographic description of QCD, Phys. Lett. B 652 (2007) 73.

[13] A. Gokalp, Y. Sarac and O. Yilmaz, Scalar $a_{0}$-meson contributions to radiative $\omega \rightarrow \pi_{0} \eta \gamma$ and $\rho_{0} \rightarrow \pi_{0} \eta \gamma$ decays, Eur. Phys. J. C 22 (2001) 327.

[14] F. De Fazio and M. R. Pennington, Probing the structure of $f_{0}(980)$ through radiative $\Phi$ decays, Phys. Lett. B 521 (2001) 15.

[15] P. Colangelo and F. De Fazio, Coupling $g_{f_{0} K^{+} K^{-}}$and the structure of $f_{0}(980)$, Phys. Lett. B 559 (2003) 49. 